\title{
The Large Magellanic Cloud Cepheids: effects of helium content variations
}

\author{
Marcella Marconi ${ }^{1}$, Roberta Carini ${ }^{2}$, Enzo Brocato $^{2}$ \\ and Gabriella Raimondo ${ }^{3}$ \\ ${ }^{1}$ INAF - Osservatorio Astronomico di Capodimonte, Via Moiariello 16, 80131 Napoli, Italy \\ email: marcella.marconi@oacn.inaf.it \\ ${ }^{2}$ INAF - Osservatorio Astronomico di Roma, via Frascati 33, 00040 Monte Porzio Catone, Italy \\ email: carini@oa-roma.inaf.it, brocato@oa-roma.inaf.it \\ ${ }^{3}$ INAF - Osservatorio Astronomico di Teramo, Mentore Maggini s.n.c., 64100 Teramo, Italy \\ email: raimondo@oa-teramo.inaf.it
}

\begin{abstract}
An extended set of evolutionary and pulsational models has been computed for two chemical compositions representative of classical Cepheids in the Large Magellanic Cloud. The comparison between the standard and He enhanced theoretical predictions is analysed and the implications for interpreting current observations and for defining the Cepheid based distance scale are discussed.
\end{abstract}

Keywords. stars: variables: Cepheids, stars: abundances, stars: distances

\section{Nonlinear convective pulsation models for LMC Cepheids: the He abundance effect}

The adopted pulsation models are nonlinear and include a nonlocal time-dependent treatment of convection (see e.g. Bono et al. 1999 and Marconi et al. 2005). A metallicity typical of LMC Cepheids, $Z=0.008$ is assumed and two He contents, $Y=0.25,0.35$ are considered (Carini et al., in preparation).

Figure 1 shows the predicted instability strip at $Z=0.008$ as a function of the adopted He abundance. Solid and dashed lines are the fundamental strip edges, whereas dotted and long-dashed lines depict the first overtone ones for the labelled $Y$ values. We notice that the instability strip gets hotter as the helium content increases at fixed $Z$, in agreement with previous results at solar metallicity (see e.g. Marconi et al. 2005). This effect reflects on the coefficients of the predicted optical Period-Luminosity (PL) relations. Indeed they are expected to get steeper as $Y$ increases from $Y=0.25$ to $Y=0.35$.

\section{Synthetic stellar populations including Cepheids}

By combining evolutionary and pulsational models (Carini et al., in preparation) we were able to produce synthetic stellar populations including Cepheids (the stars falling within the above shown instability strips) at both He contents and for various age assumptions. As an example we show in Fig. 2 the resulting populations for an age of 100 Myr. Synthetic stars falling within the predicted instability strip are represented by larger triangles. By applying the model based pulsation relation that connects the period to the mass, luminosity and effective temperature of synthetic stars falling within the instability strip, we are able to produce a period distribution for each assumption of the He content and age. Moreover, assuming a mixed population with a fraction of stars at $Y=0.25$ and the remaining part at $Y=0.35$, with a given age gap between the two subpopulations, we are able to predict the impact of the possible presence of 


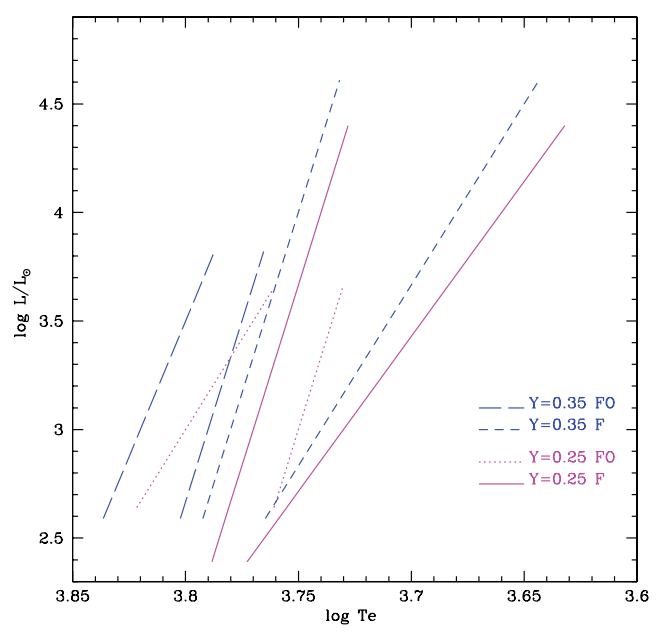

Figure 1. The predicted instability strip as a function of the adopted Y (see labels).

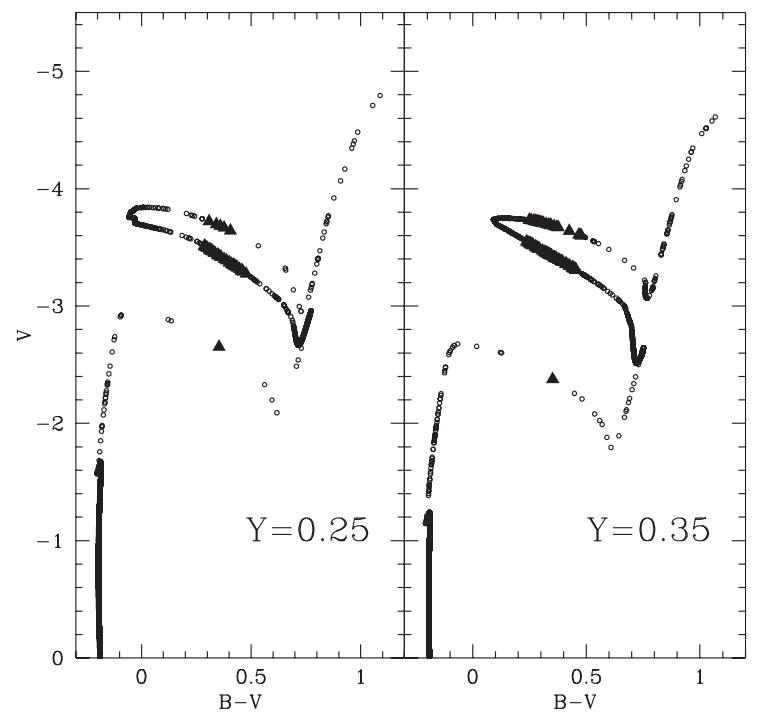

Figure 2. Synthetic stellar populations including Cepheids for an age of $100 \mathrm{Myr}$ and the two labelled He contents.

multipopulations in investigated stellar systems on the observed period histogram (see Carini et al. for details). Multiple stellar populations could be in principle observed in very populous young and intermediate-age massive clusters and star forming regions, provided that spatial resolution and distance of the objects allows reliable photometric measurements of individual stars. Current model predictions suggest that the Cepheidbased distance scale could be affected by bias due to the unexpected presence of Cepheids with huge differences in their original He content and belonging to the same stellar system for which the distance is measured.

\section{References}

Bono, G., Marconi, M., \& Stellingwerf, R. F. 1999, ApJS, 122, 167

Marconi, M., Musella, I., \& Fiorentino, G. 2005, ApJ, 632, 590 